\title{
Photoexcitation dynamics in solution-processed formamidinium lead iodide perovskite thin films for solar cell applications
}

\begin{abstract}
Hong-Hua Fang ${ }^{1}$, Feng Wang ${ }^{2}$, Sampson Adjokatse ${ }^{1}$, Ni Zhao ${ }^{2}$, Jacky Even ${ }^{3}$ and Maria Antonietta Loi ${ }^{1}$
Formamidinium lead iodide $\left(\mathrm{FAPbl}_{3}\right)$ is a newly developed hybrid perovskite that potentially can be used in high-efficiency solution-processed solar cells. Here, the temperature-dependent dynamic optical properties of three types of $\mathrm{FAPbl}_{3}$ perovskite films (fabricated using three different precursor systems) are comparatively studied. The time-resolved photoluminescence (PL) spectra reveal that $\mathrm{FAPbl}_{3}$ films made from the new precursor (a mixture of formamidinium iodide and hydrogen lead triiodide) exhibit the longest lifetime of $\mathbf{4 3 9} \mathrm{ns}$ at room temperature, suggesting a lower number of defects and lower non-radiative recombination losses compared with $\mathrm{FAPbl}_{3}$ obtained from the other two precursors. From the temperature-dependent PL spectra, a phase transition in the films is clearly observed. Meanwhile, exciton-binding energies of 8.1 and $18 \mathrm{meV}$ for the high- and lowtemperature phases are extracted, respectively. Importantly, the PL spectra for all of the samples show a single peak at room temperature, whereas at liquid-helium temperature the emission features two peaks: one in higher energy displaying a fast decay $(0.5 \mathrm{~ns})$ and a second red-shifted peak with a decay of up to several microseconds. These two emissions, separated by $\sim 18 \mathrm{meV}$, are attributed to free excitons and bound excitons with singlet and triplet characters, respectively.
\end{abstract}

Light: Science \& Applications (2016) 5, e16056; doi:10.1038/Isa.2016.56; published online 8 April 2016

Keywords: formamidinium lead iodide; low temperature; perovskite; photoluminescence; triplet exciton

\section{INTRODUCTION}

Hybrid organic-inorganic lead halide perovskites have emerged as a revolutionary class of light absorbers in solid-state solar cells ${ }^{1,2}$. The power conversion efficiency of perovskite-based solar cells has rapidly increased (currently $>20 \%$ ) due to the excellent optical and electronic properties of the perovskite material, such as superior light absorption $^{2}$, high carrier mobility and long carrier diffusion length ${ }^{3,4}$. Of the various organic-inorganic lead halide perovskite materials, methylammonium lead iodide $\left(\mathrm{MAPbI}_{3}\right)$ and its analogs are the most widely studied ${ }^{5-8}$. However, the band gaps of these materials, which range between 1.5 and $2.3 \mathrm{eV}$, are still non-optimal in terms of the optical absorption onset ${ }^{9}$. Currently, much attention is being focused on the possibility to red-shift the perovskite absorption onset to further improve the light-harvesting capability of perovskite-based solar cells ${ }^{10,11}$. An intuitive strategy involves the variation of the size of the organic cation ${ }^{12,13}$. To this end, formamidinium lead iodide $\left(\mathrm{FAPbI}_{3}\right)$, in which the relatively larger organic cation (formamidinium) replaces the methylammonium cation, is being explored because of its narrower band gap $(1.48 \mathrm{eV})$ compared with $\mathrm{MAPbI}_{3}$. $\mathrm{FAPbI}_{3}$ solar cells have been demonstrated to show a spectrally extended photocurrent generation onset and high power conversion efficiency ${ }^{14-19}$. Solar cells based on $\mathrm{FAPbI}_{3}$ materials with conversion efficiency $>20 \%$ have been recently demonstrated ${ }^{20}$. In addition, recent reports of the photophysics of $\mathrm{MAPbI}_{3}$ showed that detailed experimental studies of the excitation dynamics are not only of major importance to gain a better understanding of the photophysics of these hybrid semiconductors but also fundamental for understanding the limitation of the device performance ${ }^{8,21-23}$. In contrast to $\mathrm{MAPbI}_{3}$, knowledge of the photophysical properties of $\mathrm{FAPbI}_{3}$ is limited and thus requires further studies.

In a previous work, we fabricated $\mathrm{FAPbI}_{3}$ perovskite solar cells using three different precursor combinations: (i) a mixture of formamidinium iodide (FAI) and hydrogen lead triiodide $\left(\mathrm{HPbI}_{3}\right)\left(\mathrm{FAI} / \mathrm{HPbI}_{3}\right)$; (ii) a mixture of FAI and $\mathrm{PbI}_{2}\left(\mathrm{FAI} / \mathrm{PbI}_{2}\right)$; and (iii) $\mathrm{FAI} / \mathrm{PbI}_{2}$ with hydrogen acid $(\mathrm{HI})$ additive $\left(\mathrm{FAI} / \mathrm{PbI}_{2}+\mathrm{HI}\right)^{16}$. Compared with the commonly used precursors $\left(\mathrm{FAI} / \mathrm{PbI}_{2}\right.$ or $\mathrm{FAI} / \mathrm{PbI}_{2}$ with $\mathrm{HI}$ ), the devices based on the newly synthesized precursor $\left(\mathrm{FAI} / \mathrm{HPbI}_{3}\right)$ showed improved morphology and a much higher average efficiency up to $15.4 \%$. Such a dependence on the precursor used to synthesize the material appears to be non-trivial and calls for a systematic investigation of the optical properties of the $\mathrm{FAPbI}_{3}$ obtained from the different precursors.

${ }^{1}$ Photophysics \& OptoElectronics, Zernike Institute for Advanced Materials, Nijenborgh 4, Groningen 9747 AG, The Netherlands; ${ }^{2}$ Department of Electronic Engineering, The Chinese University of Hong Kong, New Territories, Hong Kong and ${ }^{3}$ Université Européenne de Bretagne, INSA, FOTON, UMR 6082, 35708 Rennes, France

Correspondence: MA Loi, Email: m.a.loi@rug.nl; N Zhao, Email: nzhao@ee.cuhk.edu.hk; J Even, Email: Jacky.Even@insa-rennes.fr

Received 11 September 2015; revised 25 November 2015; accepted 1 December 2015; accepted article preview online 25 December 2015 
In this report, the temperature-dependent optical properties of $\mathrm{FAPbI}_{3}$ perovskite films based on different precursor systems (FAI/ $\mathrm{HPbI}_{3}, \mathrm{FAI} / \mathrm{PbI}_{2}$ and $\mathrm{FAI} / \mathrm{PbI}_{2}+\mathrm{HI}$ ) are comparatively investigated. By using temperature-dependent optical spectroscopy, their excitonbinding energies for different crystal phases are extracted. At room temperature, we found the lifetimes to vary from tens to hundreds of nanoseconds, and the photoluminescence (PL) spectra for all of the samples show a single peak. At liquid-helium temperature, the emission features two peaks, one in the blue region that exhibits a rapid decay $(0.5 \mathrm{~ns})$ and a second red-shifted peak with a decay of up to several microseconds. These two emissions, separated by $\sim 18 \mathrm{meV}$, are attributed to free excitons (FEs) and bound excitons (BEs), with singlet and triplet characters, respectively.

\section{MATERIALS AND METHODS}

Perovskite films were prepared according to a previous report ${ }^{16}$. We noted that the formation process of $\mathrm{FAPbI}_{3}$ perovskite is markedly different when using different precursor combinations. To optimize the structural quality of the perovskite films, different procedures were adopted for different precursors. The annealing time was optimized by controlling the formation of the perovskite. For $\mathrm{FAI} / \mathrm{PbI}_{2}$-based thin films, FAI and $\mathrm{PbI}_{2}$ were dissolved in anhydrous N,N-dimethylformamide at a molar ratio of 1:1. After spin coating at 3000 r.p.m. for $60 \mathrm{~s}$ on a glass substrate and then drying at $160^{\circ} \mathrm{C}$ for $40 \mathrm{~min}$, perovskite films were formed. The fabrication of $\mathrm{FAPbI}_{3}$ films from $\mathrm{FAI} / \mathrm{PbI}_{2}+\mathrm{HI}$ was similar to that of $\mathrm{FAPbI}_{3}$ film made from $\mathrm{FAI} / \mathrm{PbI}_{2}$, except 5 vol\% hydriodic acid was added to the FAI and $\mathrm{PbI}_{2}$ solution, and the annealing time was $30 \mathrm{~min}$. Similarly, $\mathrm{FAPbI}_{3}$ films fabricated from new precursors were prepared by mixing $\mathrm{FAI}$ and $\mathrm{HPbI}_{3}$, and the annealing time was increased to $80 \mathrm{~min}$. Before performing the PL measurements, all films were sealed using a blank glass and epoxy adhesive (Epo-Tek OG 159-2, Nivelles, Belgium) to prevent degradation of the perovskite. Adhesive was solidified under UV irradiation.

PL measurements were performed by exciting the samples with the second harmonic $(400 \mathrm{~nm})$ of a mode-locked Ti:sapphire laser (Mira 900, Coherent, Santa Clara, CA, USA). The laser power at the sample was adjusted using neutral density filters. The excitation beam was spatially limited by an iris and then focused with a $150-\mathrm{mm}$ focal length lens. Fluorescence was collected into a spectrometer equipped with a 50 lines per $\mathrm{mm}$ grating and then recorded using an Image EM CCD camera from (Hamamatsu, Japan). The spectra were corrected for the spectral response of the set up. Time-resolved traces were recorded using a Hamamatsu streak camera working in single sweep mode. The excitation source was the same mode-locked femtosecond laser with a repetition rate of $76 \mathrm{MHz}$; a pulse picker was inserted in the optical path to decrease the repetition rate of the laser pulses when needed. For the low-temperature measurements, a liquid-helium continuous flow optical cryostat (Optistat, Oxford, UK) was used.

Confocal laser scanning microscopy measurements were performed using a Nikon (Tokyo, Japan) Eclipse Ti microscope in the backscattering configuration. A Nikon $\times 40$ Plan Apo objective lens was used for all the images reported. The excitation source was an Ar+ laser (excitation wavelength at $488 \mathrm{~nm}$ ). The PL images of the samples were recorded by reconstructing the PL intensity point by point.

\section{RESULTS AND DISCUSSION}

Figure 1a-1c shows confocal laser scanning microscope images of the films fabricated from $\mathrm{FAI} / \mathrm{PbI}_{2}, \mathrm{FAI} / \mathrm{PbI}_{2}+\mathrm{HI}$ and $\mathrm{FAI} / \mathrm{HPbI}_{3}$ precursors, respectively. The $x-y$ spatial resolution of the images is estimated to be $\sim 300 \mathrm{~nm}$. The $\mathrm{FAPbI}_{3}$ films synthesized using FAI/ $\mathrm{HPbI}_{3}$ and $\mathrm{FAI} / \mathrm{PbI}_{2}+\mathrm{HI}$ have a much brighter appearance than the
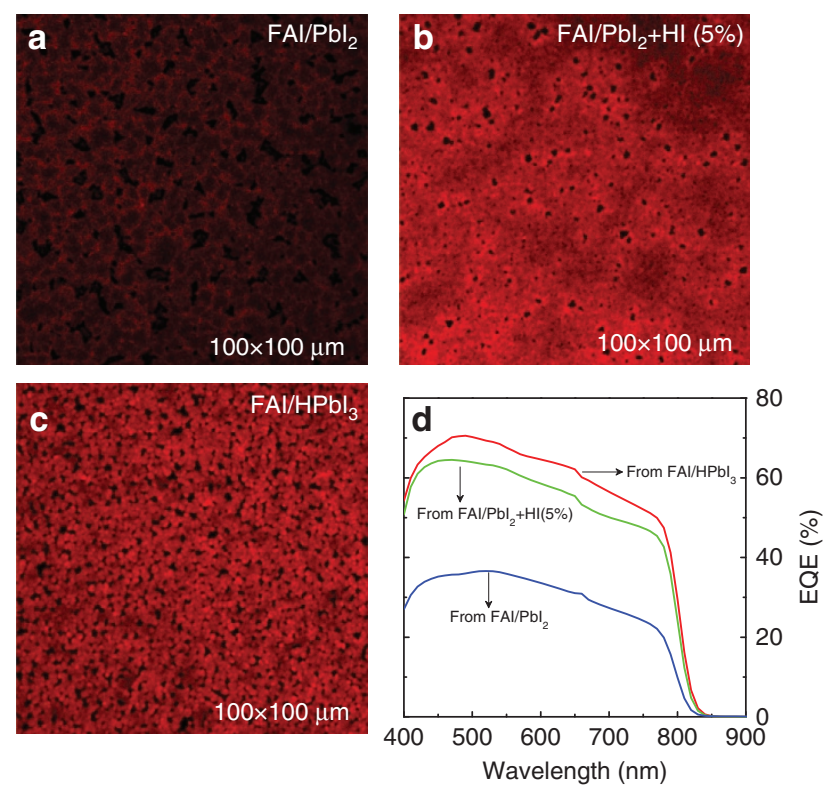

Figure 1 Confocal laser scanning microscopy of $\mathrm{FAPbl}_{3}$ films prepared from different precursors. (a) $\mathrm{FAl} / \mathrm{Pbl}_{2}$, (b) $\mathrm{FAl} / \mathrm{Pbl}_{2}$ with $5 \% \mathrm{HI}$ and (c) $\mathrm{FAl} / \mathrm{HPbl}_{3}$. (d) $\mathrm{EQE}$ spectra of $\mathrm{FAPbl}_{3}$ solar cells obtained from the three different precursors. EQE, external quantum efficiency.

film fabricated from $\mathrm{FAI} / \mathrm{PbI}_{2}$, suggesting their higher quantum efficiency. Furthermore, the $\left(\mathrm{FAI} / \mathrm{PbI}_{2}+\mathrm{HI}\right)$-based film exhibits the most uniform morphology followed by the $\mathrm{FAI} / \mathrm{HPbI}_{3}$ film. Thin films formed from the $\mathrm{FAI} / \mathrm{HPbI}_{3}$ precursor solution exhibit small-size domains, probably indicating the higher crystallinity of this sample compared with the other two. The spectrally resolved external quantum efficiencies shown in Figure 1d show a substantially higher efficiency for the $\mathrm{FAI} / \mathrm{HPbI}_{3}$-based solar cells followed by that using $\mathrm{FAI} / \mathrm{PbI}_{2}+\mathrm{HI}$ as the active layer.

The room-temperature PL spectra of the three different $\mathrm{FAPbI}_{3}$ perovskite films are shown in Figure 2a. Their PL peaks are centered at $\sim 820 \mathrm{~nm}$ with a full-width at half-maximum (FWHM) of $\sim 42 \mathrm{~nm}$, consistent with previously reported data ${ }^{18}$. Notably, the emission peak of $\mathrm{FAI} / \mathrm{HPbI}_{3}$-based films shows a $5-6 \mathrm{~nm}$ blue-shift compared with the other two films, probably because of the low trap density of FAI/ $\mathrm{HPbI}_{3}$-based films. It has recently been demonstrated that measurements of the PL dynamics yield important information regarding the diffusion length of the photoexcited species in the devices, with longer PL lifetimes indirectly indicating longer charge diffusion lengths ${ }^{3}$. Figure $2 \mathrm{~b}$ illustrates the decay time of the three samples at room temperature. $\mathrm{FAI} / \mathrm{HPbI}_{3}$-based films display an unusually long decay that can be fitted with a mono-exponential function with a lifetime of $439 \mathrm{~ns}$. The lifetime extracted for $\left(\mathrm{FAI} / \mathrm{PbI}_{2}+\mathrm{HI}\right)$ - and $\mathrm{FAI} / \mathrm{PbI}_{2}$-based films are 134 and $95 \mathrm{~ns}$, respectively. In these materials, the lifetime of the photoexcited species are sufficiently long to enable diffusion to the charge selective contacts where they are efficiently extracted; thus, a large fraction of the solar spectrum can be collected.

Efficient PL emission and long lifetime can be considered an indicator of low recombination losses in solar cells. As demonstrated in previous studies, the $\mathrm{FAI} / \mathrm{HPbI}_{3}$-based perovskite solar cells exhibit high external quantum efficiency ${ }^{16}$. The $\mathrm{FAI} / \mathrm{HPbI}_{3}$-based film exhibits the longest emission lifetime, explaining why it results in the best performance among the three different precursor-based systems. Figure $2 \mathrm{c}$ shows the two-dimensional contour plot of a typical timeresolved $\mathrm{PL}$ spectrum of a $\mathrm{FAI} / \mathrm{HPbI}_{3}$ thin film, from which we can 
a

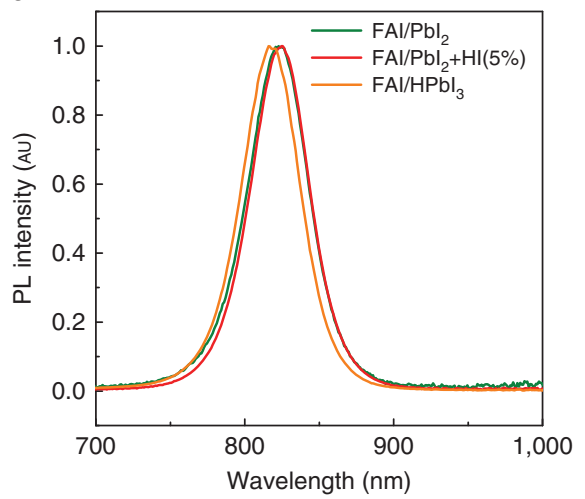

b

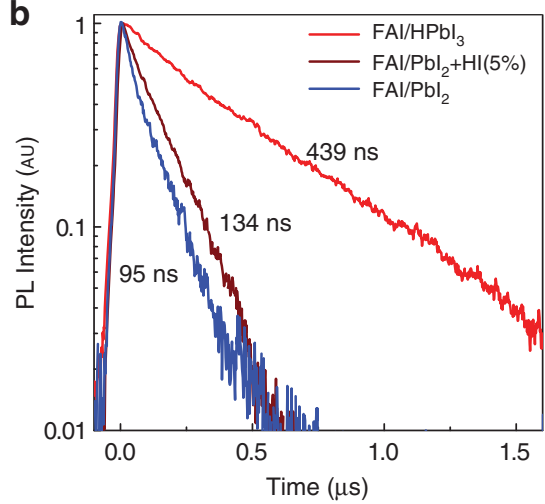

C
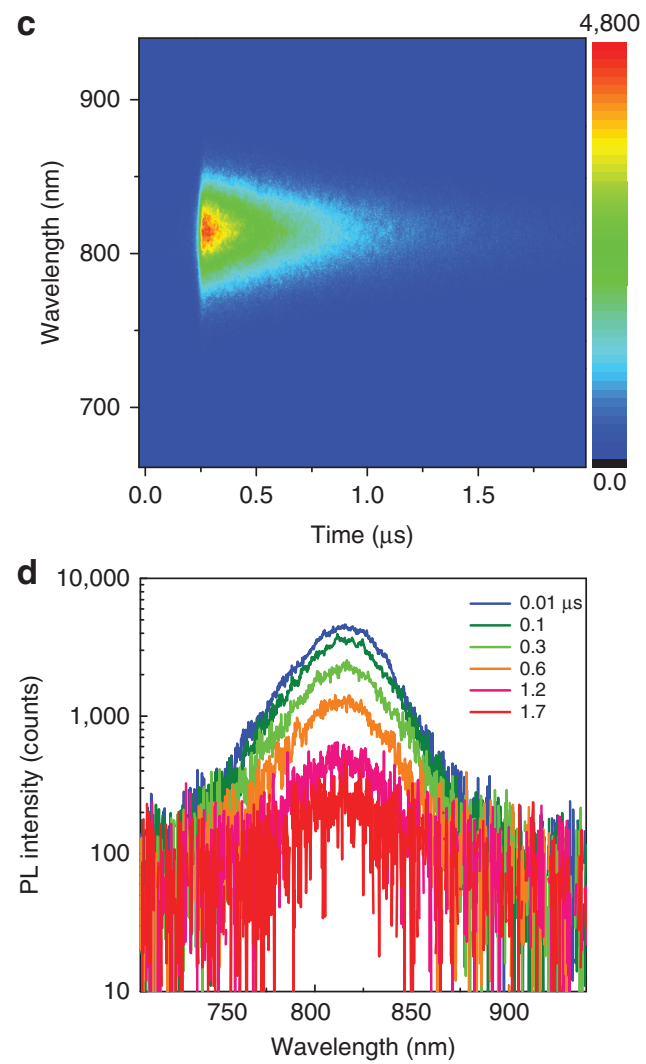

Figure 2 (a) PL spectra of $\mathrm{FAPbl}_{3}$ films prepared from different precursor systems. (b) Time-resolved PL decay at room temperature. (c) 2D pseudo-color plot of streak camera data from a FAl/HPbl 3 -based film. (d) Log-plot of PL spectra at different times after photoexcitation. 2D, two-dimensional.
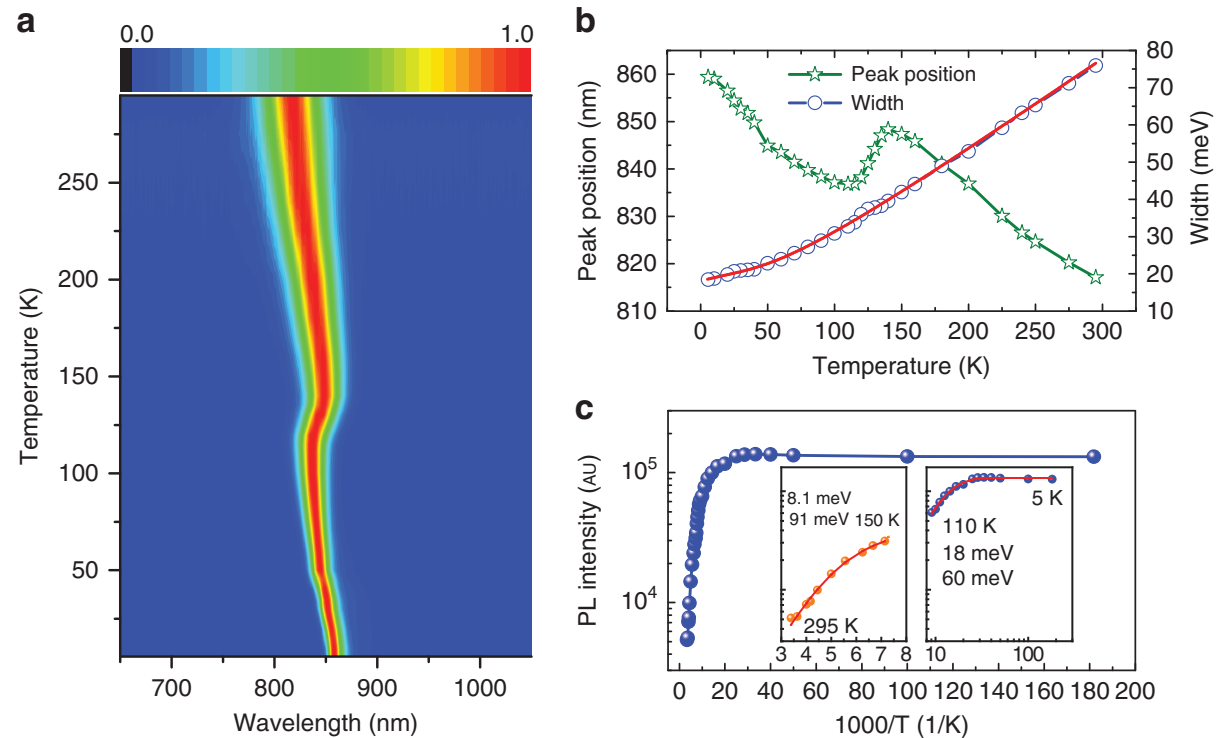

Figure 3 (a) 2D pseudo-color plot of the normalized emission spectra under a pump fluence of $1.4 \mu \mathrm{J} \mathrm{cm}^{-2}$ at different temperatures; the PL spectra are normalized at their maximum intensity. (b) Peak position and FWHM of the PL spectra as a function of temperature. The red solid line is the result of a fit, including contributions from inhomogeneous broadening, acoustic and optical phonons. (c) Temperature-dependent data of the integrated PL intensity. Inset: the fitted curve from 295 to $150 \mathrm{~K}$ and from 110 to $5 \mathrm{~K}$.

extract emission spectra at different times after excitation. It is evident that the shape of the spectrum is independent of the time elapsed after excitation, as shown in Figure 2d. Similar behavior is displayed by the $\left(\mathrm{FAI} / \mathrm{PbI}_{2}+\mathrm{HI}\right)$ - and $\mathrm{FAI} / \mathrm{PbI}_{2}$-based films.
To investigate the origin of the enhanced lifetime of the FAI/HPbI perovskite film, the PL properties at different temperatures were investigated. Figure $3 \mathrm{a}$ shows the temperature evolution of the PL spectra for the $\mathrm{FAI} / \mathrm{HPbI}_{3}$ film under an excitation power density of 
$1.4 \mu \mathrm{Jm}^{-2}$. The spectra are normalized using the maximum PL intensity at each temperature. A clear evolution of the peak position is revealed in the contour plot of the PL spectra at the different temperatures. For the other two precursor-based samples, the temperature-dependent PL (Supplementary Fig. S1) shows a similar behavior to the one of the $\mathrm{FAI} / \mathrm{HPbI}_{3}$-based film. The PL spectrum shows a monotonous red-shift towards $848 \mathrm{~nm}$ to $\sim 140 \mathrm{~K}$ (Figure 3a). This red-shift is followed by a sudden blue-shift of the PL peak from 848 to $836 \mathrm{~nm}$ between 140 and $120 \mathrm{~K}$, and then a new monotonous red-shift continues to $859 \mathrm{~nm}$ at $5 \mathrm{~K}$. This red-shift is accompanied by a progressive reduction of the FWHM, as shown in Figure $3 \mathrm{~b}$. The width reduces from $76 \mathrm{meV}$ (i.e., $41 \mathrm{~nm}$ in wavelength) at $295 \mathrm{~K}$ to $18 \mathrm{meV}(12 \mathrm{~nm})$ at $5 \mathrm{~K}$. The abrupt variation $(20 \mathrm{meV}$, i.e., from $847.5 \mathrm{~nm}$ to $836 \mathrm{~nm}$ ) of the exciton energy around $140 \mathrm{~K}$, is significantly smaller than the $90-100 \mathrm{meV}$ value that we and others have reported for $\mathrm{MAPbI}_{3}$ at close to $T=163 \mathrm{~K}^{21,23}$. In $\mathrm{MAPbI}_{3}$, the variation of peak position is related to a sharp first-order structural phase transition between the $\beta$ tetragonal phase $(\mathrm{I} 4 / \mathrm{mcm})$ and the $\gamma$ orthorhombic phase (Pnma) due to the lack of a group-subgroup relationship between the space groups. The structural phase transition corresponds to a change of the perovskite tilt system, coupled with orientational disorder of all the MA cations appearing in the $\beta$ phase. The peak position variation shown in Figure $3 \mathrm{~b}$ for $\mathrm{FAPbI}_{3}$ is in good agreement with the $\alpha-\beta$ transition temperature reported by X-ray diffraction $^{24}$. The structures of the $\alpha$ and $\beta$ phase of $\mathrm{FAPbI}_{3}$ are trigonal, $\mathrm{P} 3 \mathrm{~m} 1(Z=3)$ and $\mathrm{P} 3(Z=12)$, respectively. The $\mathrm{P} 3 \mathrm{~m} 1$ phase of $\mathrm{FAPbI}_{3}$ was referred to as the $\alpha$ phase; however, the authors were uncertain whether there is yet another higher symmetry phase at higher temperatures ${ }^{24}$. The $\mathrm{P} 3 \mathrm{ml}$ trigonal phase exhibits complete orientational disorder of the cations and a very small cell distortion compared with the Pm-3m high-temperature cubic phase of $\mathrm{MAPbI}_{3}$. The reduced exciton energy shift (Figure $3 \mathrm{~b}$ ) of $\mathrm{FAPbI}_{3}$ compared with $\mathrm{MAPbI}_{3}$, appears to indicate that the $\alpha-\beta$ transition in $\mathrm{FAPbI}_{3}$ exhibits a less-pronounced first-order character than the $\beta-\gamma$ transition in $\mathrm{MAPbI}_{3}$ at $T=163 \mathrm{~K}$. This observation is consistent with the crystallographic group-subgroup relationship between the $\alpha$ and $\beta$ phases of $\mathrm{FAPbI}_{3}{ }^{24}$, the transition being thus defined as a weak first-order phase transition. Only a minor fraction of the FA cations retain an orientational disorder in the $\beta$ phase. This behavior may be attributed to the geometry and symmetry of the FA cation, which is more flexible to comply with the lattice distortions ${ }^{24}$. The existence of a completely ordered low-temperature monoclinic $\gamma$ phase was postulated from the inspection of X-ray diffraction data at $\sim 100 \mathrm{~K}^{24}$, but the structure was not determined. However, the results obtained in this work do not indicate that a possible $\beta-\gamma$ transition in $\mathrm{FAPbI}_{3}$ occurs at low temperature. The possible existence of a $\beta-\gamma$ phase transition in $\mathrm{FAPbI}_{3}$ at low temperature deserves additional experimental studies. The absence of a spectroscopic signature between 150 and $300 \mathrm{~K}$ is consistent with the stability of the $\alpha$ phase in this temperature range up to room temperature.

The exciton-binding energy is a key parameter for hybrid perovskites; however, the precise binding energy is not clearly known. The reported values for $\mathrm{MAPbI}_{3}$ perovskite covers a broad range, from several $\mathrm{meV}$ to tens of $\mathrm{meV}^{22,25-27}$. For example, a binding energy of $62.3 \mathrm{meV}$ for $\mathrm{CH}_{3} \mathrm{NH}_{3} \mathrm{PbI}_{3-\mathrm{x}} \mathrm{Cl}_{\mathrm{x}}$ was extracted by Wu et al. ${ }^{28}$ based on an analysis of the thermal quenching of the PL intensity. Recently, an increasing number of reports have suggested that a lower binding energy exists in perovskites. Even et al. predicted that the binding energy is much lower and undergoes a further reduction from 15 to $5 \mathrm{meV}$ above the $\beta-\gamma$ transition because excitons are strongly screened by disordered cation orientations, as well as vibrations of the inorganic lattice at room temperature. In good agreement with this prediction, Miyata et al. reported the exciton-binding energy of $\mathrm{CH}_{3} \mathrm{NH}_{3} \mathrm{PbI}_{3}$ to be reduced from 16 to $6 \mathrm{meV}$ at $300 \mathrm{~K}$ when measured by the magneto-absorption technique ${ }^{23}$. This discrepancy between the PL and magneto-absorption methods may be due to additional thermal quenching processes, which are not taken into account when using temperature-dependent PL to estimate the binding energy ${ }^{29}$. If several processes contribute, then additional exponential terms can be added with further activation energies ${ }^{30}$.

In our experiment, we observed that the PL intensity of the $\mathrm{FAPbI}_{3}$ thin film was quenched when the temperature increases from 5 to $295 \mathrm{~K}$. As discussed previously, there is a $\alpha-\beta$ transition modifying the emission properties at $\sim 150 \mathrm{~K}$. To exclude the phase transition effect, the temperature-dependent PL emission intensity from 150 to $295 \mathrm{~K}$ is fitted. Instead of single-activation energy, the intensity quenching is described by a thermally activated process with two activation energies and is assumed to have the following form:

$$
I_{T}=\frac{I_{0}}{1+A \exp \left(\frac{-E_{\mathrm{a}}}{k_{\mathrm{B}} T}\right)+B \exp \left(\frac{-E_{\mathrm{b}}}{k_{\mathrm{B}} T}\right)}
$$

where $I_{0}$ is the emission intensity extrapolated at $0 \mathrm{~K}, E_{\mathrm{a}}$ and $E_{\mathrm{b}}$ are the activation energies, and $k_{\mathrm{B}}$ is the Boltzmann constant. Figure $3 \mathrm{c}$ shows the integrated PL intensity versus temperature, where the hightemperature phase and low-temperature phase are fitted separately. On the basis of the least-squares fit, the $E_{\mathrm{a}}$ and $E_{\mathrm{b}}$ activation energies are estimated to be 8.1 and $91 \mathrm{meV}$, respectively, for the $\mathrm{HPbI}_{3}$-based film and 9.9 and $113 \mathrm{meV}$, respectively, for the $\mathrm{FAI} / \mathrm{PbI}_{2}$ sample. The smaller activation energy is comparable with the room-temperature binding energy $(6 \mathrm{meV})$ in $\mathrm{MAPbI}_{3}$ estimated by magneto-absorption. Miyata et al. demonstrated that in $\mathrm{MAPbI}_{3}$, the exciton-binding energy is equal to $6 \mathrm{meV}$ at room temperature, but increases to $16 \mathrm{meV}$ below the $\beta-\gamma$ transition. By fitting the PL intensity from 5 to $110 \mathrm{~K}$, we found that the smaller activation energy increases from 8.1 to $18 \mathrm{meV}$ in $\mathrm{HPbI}_{3}$ films. Compared with $\mathrm{MAPbI}_{3}$, this result also suggests that the exciton-binding energy is reduced when the cations are totally disordered in the $\alpha$ phase.

To study the exciton - phonon coupling effect, we analyzed the temperature dependence of the PL broadening obtained from the Gaussian de-convolution. The experimental values of the PL spectrum FWHM (Figure 3b), obtained from a Gaussian best fit of the spectra clearly show that the FWHM increases with temperature. At a temperature of $5 \mathrm{~K}$, the spectrum peak is at $\sim 860 \mathrm{~nm}$ with an asymmetric shape and a low-energy tail (Supplementary Fig. S2). The spectra change into a Boltzmann-like shape with increasing temperature (Figure 4a). The PL line width also broadens from 18.5 to $76 \mathrm{meV}$ with increasing temperature from 5 to $295 \mathrm{~K}$. We fitted the experimental FWHM to the following classical equation ${ }^{31,32}$ :

$$
\Gamma(T)=\Gamma_{\mathrm{inh}}+\sigma T+\frac{\Gamma_{\mathrm{LO}}}{e^{h \omega_{\mathrm{o}} / k_{\mathrm{B}} T}-1}
$$

Here, $\Gamma_{\text {inh }}$ is the inhomogeneous broadening contribution, which is temperature independent, $\sigma$ and $\Gamma_{\mathrm{LO}}$ are the exciton-acoustic phonon interaction and the exciton - optical phonon coupling coefficient, respectively, $h \omega_{\mathrm{o}}$ is an optical phonon energy, and $k_{\mathrm{B}}$ is the Boltzmann constant. As the temperature increases, the acoustic and optical phonon contributions dominate and the line width increases with temperature. From the best fit, the exciton-acoustic phonon coupling constant $\sigma$ is $\sim 78 \mu \mathrm{eV} \mathrm{K}^{-1}$. The inhomogeneous broadening contribution is $18.1 \mathrm{meV}$. The best-fit value of the exciton-optical phonon-coupling coefficient is $34.8 \mathrm{meV}$, and the optical phonon 
energy is $18 \mathrm{meV}$. This latter value is assumed to be the effective average because the $\mathrm{FAPbI}_{3}$ lattice possesses more than one optical phonon.

To better understand the photoexcitation process, the dynamics of the emission processes at different temperatures were investigated under femtosecond pulsed photoexcitation. Figure $4 \mathrm{a}$ shows PL spectra at selected temperatures and corresponding time resolved
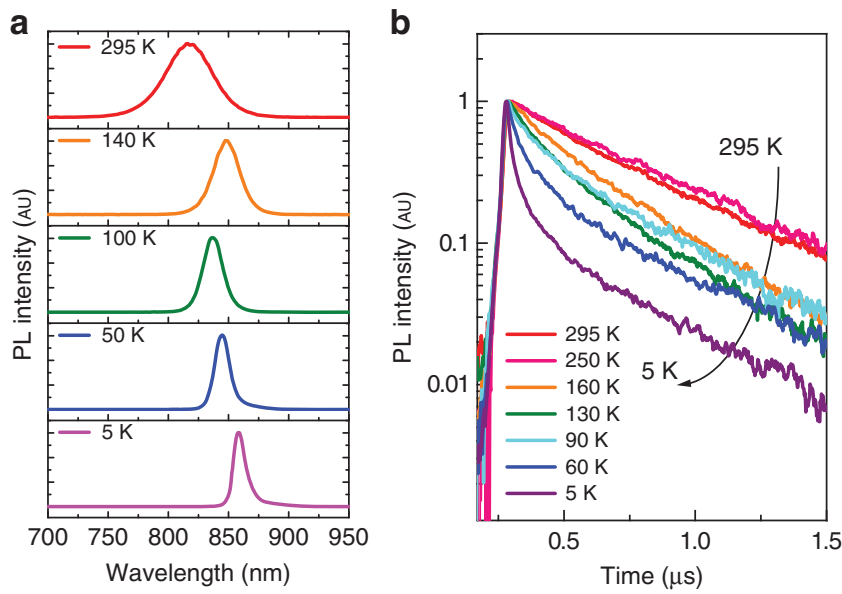

Figure 4 (a) PL spectra and (b) TRPL decay kinetics after photoexcitation with an excitation power density of $1.4 \mu \mathrm{J} \mathrm{cm}^{-2}$ at representative temperatures. photoluminescence (TRPL) decay kinetics after photoexcitation, with excitation power density of $1.4 \mu \mathrm{J} \mathrm{cm}^{-2}$. At room temperature, the emission decay is mono-exponential, with a lifetime of $439 \mathrm{~ns}$, and the emission peak is invariant with time, as already shown in Figure 2. At low temperature, the emission decay is no longer monoexponential. In addition to the slow emission decay, an ultrafast decay is observed when the temperature is decreased, as clearly shown in Figure $4 b$.

Unlike the mono-exponential single-peak emission at room temperature, an extremely fast emission decay at the blue band $(859 \mathrm{~nm})$ and a long-lived emission at the red band $(870 \mathrm{~nm})$ are observed at $5 \mathrm{~K}$ (Figure 5a). This observation is similar to the behavior we have recently reported in $\mathrm{MAPbI}_{3}$ single crystals at liquid-helium temperature. For the purposes of discussion, we refer to the fast emission decay as PL I and the slow emission as PL II. The PL II and PL I emissions are separated by an energy gap $\Delta \mathrm{E}$. We use two methods to determine the value of $\Delta \mathrm{E}$ : one method is based on the spectra taken at different delay times after photoexcitation (Figure 5b), whereas the other method is based on fitting the steady-state PL. Using both methods, $\Delta \mathrm{E}$ of $18 \mathrm{meV}$ is obtained. We use a simple three-level model to interpret the data. The energy level diagram illustrating the model is presented in Figure $5 c$, where the levels include a ground state, a short-lived upper state with lifetime $t_{1}$ and a long-lived lower state with lifetime $t_{2}$. The lifetime of the short-lived upper state is $0.5 \mathrm{~ns}$, whereas that for the long-lived state is up to $2.6 \mu \mathrm{s}$. Figure $5 \mathrm{~d}$ shows the time-resolved PL curve in the case of the PL II at different power. The decay time is shortened at high-power intensity, which
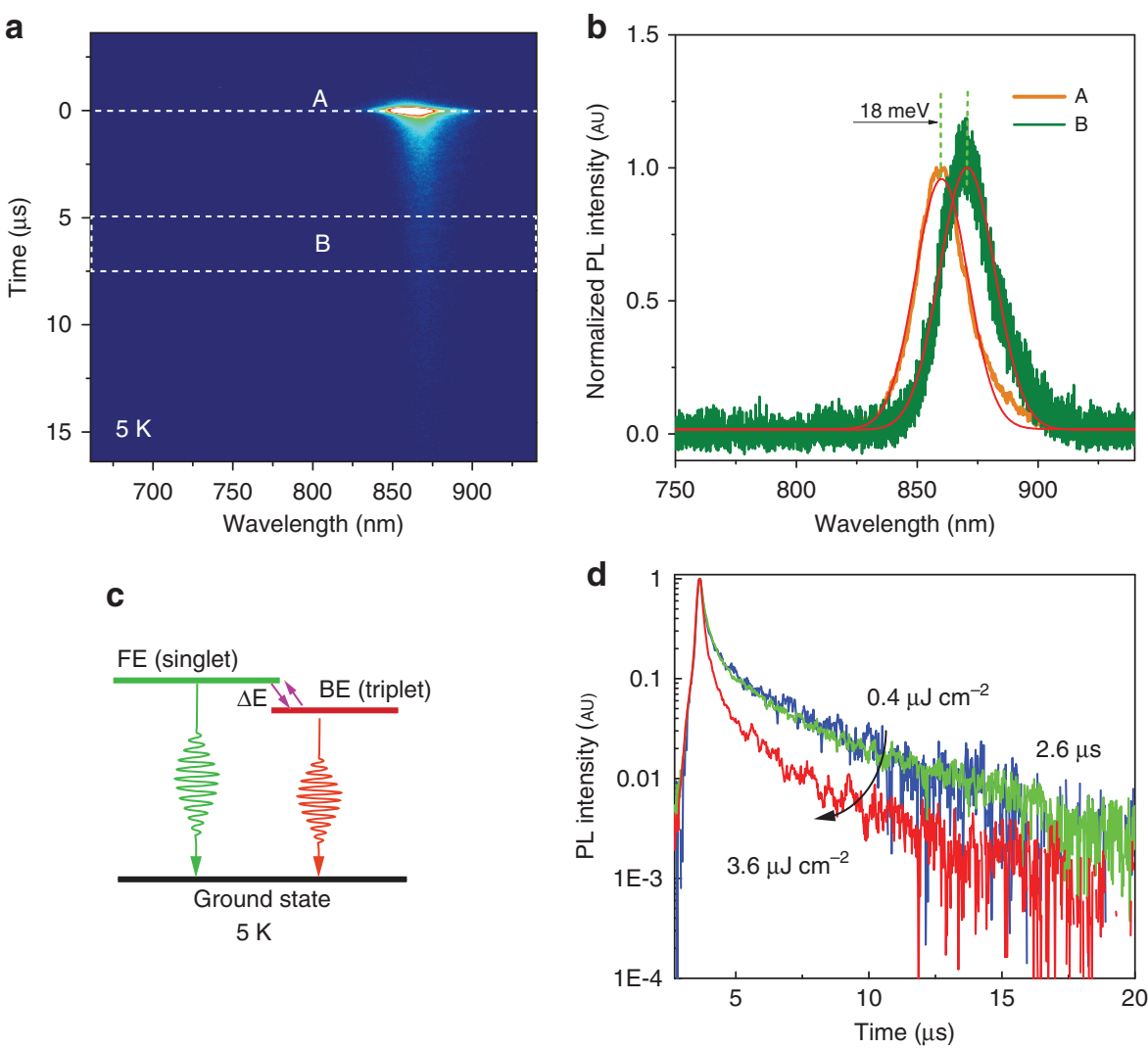

Figure 5 (a) Streak camera data showing the time-resolved PL emission at $5 \mathrm{~K}$. (b) PL spectra (with Gaussian fit) taken at different time delays after excitation. $\mathrm{A}$ is the PL spectrum at time $=0 ; \mathrm{B}$ is the PL spectrum taken from 5 to $7.5 \mu \mathrm{s}$. (c) Proposed energy level diagram for FAPbl3 perovskite at $5 \mathrm{~K}$, involving a $\mathrm{FE}$ and a $\mathrm{BE}$. (d) Power dependence of the time-resolved $\mathrm{PL}$ curve. BE, bound exciton; $\mathrm{FAPb} \mathrm{F}_{3}$, formamidinium lead iodide; $\mathrm{FE}$, free exciton; $\mathrm{PL}$, photoluminescence. 
might be related to a saturation effect at high density excitation ${ }^{21}$. Note that PL II and PL I are observed for all of the samples at low temperature, with a very small variation in the separation of the two peaks (from 15 to $18 \mathrm{meV}$, see Supplementary Fig. S3 in Supplementary Information).

The possible origins of the two radiative transitions appearing at low temperature are listed below:

(1) A band edge transition (PL I) and a FE (PL II);

(2) A band edge transition (PL I) and a BE (PL II);

(3) A FE (PL I) and a longitudinal optical phonon replica (PL II);

(4) A singlet (PL I) - triplet (PL II) splitting of the same exciton line;

(5) A FE (PL I) and a BE with a possible triplet character (similar to $\left.\mathrm{MAPbI}_{3}\right)^{21}$.

The reader may refer to similar extensive discussions for both the three-dimensional (3D) $\mathrm{MAPbI}_{3}$ hybrid perovskite and the $\left(\mathrm{C}_{6} \mathrm{H}_{5} \mathrm{C}_{2} \mathrm{H}_{4}-\mathrm{NH}_{3}\right)_{2}-\mathrm{PbI}_{4}$ (PEPI) layered hybrid perovskite ${ }^{21,31}$. The first hypothesis appears to be unlikely because PL II corresponds to a long-lived state. The second case may account for the long lifetime for PL II; however, the small BE-binding energy of $18 \mathrm{meV}$ almost corresponds to the FE-binding energy deduced at higher temperature. The third case should be considered carefully because the PL I to PL II energy splitting is on the order of the optical phonon energy of 3D hybrid perovskites, contrary to $\mathrm{MAPbI}_{3}$, where the same splitting amounts to $\sim 33 \mathrm{meV}$. Following a similar discussion for the PEPI compound ${ }^{31}$, we note that the PL I and PL II peaks have very different lifetimes as well as very different power dependences. The emission peak is blue-shifted as the excitation power is increased (Supplementary Fig. S4). The phonon replica hypothesis can thus be ruled out because similar behaviors would be expected for the two peaks. The fourth case may be excluded on the basis of a theoretical prediction of the singlet-triplet splitting value in these materials $(2 \mathrm{meV})^{33}$, which appears much smaller than the observed PL I to PL II energy splitting $(18 \mathrm{meV})$. The last case appears to be the most plausible, although one may wonder why the FE-BE energy separation is smaller in $\mathrm{FAPbI}_{3}$ than in $\mathrm{MAPbI}_{3}{ }^{21}$. This difference can be attributed to the fact that the BE state involves an exciton strongly bound to the surrounding organic cations. The differences between the FA and the MA cations are therefore very important for BE states. As previously shown, the abrupt variation of the exciton energy at the $\alpha-\beta$ transition is $\sim 4-5$ times smaller in $\mathrm{FAPbI}_{3}$ than for the $\beta-\gamma$ transition of $\mathrm{MAPbI}_{3}$. This result is attributed to the fact that the FA cation is much more flexible than the MA cation, leading to reduced lattice distortions. Such distortions may appear close to defects or grain boundaries; however, we can also speculate that in $\mathrm{FAPbI}_{3}$, as in $\mathrm{MAPbI}_{3}{ }^{21}$, the surface of the crystal is the main location of the traps.

\section{CONCLUSIONS}

In summary, we studied the photogeneration and recombination of charge carriers in $\mathrm{FAPbI}_{3}$ thin films. The lifetime of the photoexcitation in the thin films at room temperature was found to vary from tens to hundreds of nanoseconds, depending on which precursor is used. A strong relationship between the photoexcitation dynamics and morphologies of the thin films was observed. The film starting from the new precursor $\mathrm{HPbI}_{3}$ exhibited a much longer lifetime (up to $439 \mathrm{~ns}$ ) than the other two, explaining why the solar cells exhibited the highest average performance. Interestingly, at low temperature, the samples share common features: a fast emission decay (PL I) in the blue region and a very long-lived red-shifted emission decay (PL II) up to several microseconds, which are attributed to the FE and the BE, respectively. The lifetime for PL I is $<1 \mathrm{~ns}$, whereas it is $2.6 \mu \mathrm{s}$ for the PL II emission, three orders of magnitude longer than that of PL I, exhibiting a possible triplet character. The results presented provide important fundamental insights into the photophysics of hybrid perovskites. Our results provide crucial information regarding the photophysics of these novel hybrid perovskite materials, which will in turn enable improvements in solar cell performance and a better understanding of their optoelectronic properties.

\section{ACKNOWLEDGEMENTS}

The work in Groningen was partially supported by the European Research Council, ERC Starting Grant (No. 306983) 'Hybrid solution processable materials for opto-electronic devices' (ERC-HySPOD) and by the Foundation for Fundamental Research on Matter (FOM), which is part of the Netherlands Organization for Scientific Research (NWO), under the framework of the FOM Focus Group 'Next Generation Organic Photovoltaics'. J Even's work is supported by the Fondation d'entreprises banque Populaire de l'Ouest under Grant PEROPHOT 2015. The technical support of A Kamp and J Kuiper is highly appreciated. We thank TTM Palstra for insightful discussions.

1 Lee MM, Teuscher J, Miyasaka T, Murakami TN, Snaith HJ. Efficient hybrid solar cells based on meso-superstructured organometal halide perovskites. Science 2012; 338: 643-647.

2 Green MA, Ho-Baillie A, Snaith HJ. The emergence of perovskite solar cells. Nat Photon 2014; 8: 506-514.

3 Stranks SD, Eperon GE, Grancini G, Menelaou C, Alcocer MJP et al. Electron-hole diffusion lengths exceeding 1 micrometer in an organometal trihalide perovskite absorber. Science 2013; 342: 341-344.

4 Dong QF, Fang YJ, Shao YC, Mulligan P, Qiu J et al. Electron-hole diffusion lengths $>175 \mu \mathrm{m}$ in solution grown $\mathrm{CH}_{3} \mathrm{NH}_{3} \mathrm{Pbl}_{3}$ single crystals. Science 2015; 347 : 967-970.

5 Liu MZ, Johnston MB, Snaith HJ. Efficient planar heterojunction perovskite solar cells by vapour deposition. Nature 2013; 501: 395-398.

6 Choi JJ, Yang XH, Norman ZM, Billinge SJL, Owen JS. Structure of methylammonium lead iodide within mesoporous titanium dioxide: active material in high-performance perovskite solar cells. Nano Lett 2013; 14: 127-133.

7 Saba M, Cadelano M, Marongiu D, Chen F, Sarritzu V et al. Correlated electron-hole plasma in organometal perovskites. Nat Commun 2014; 5: 5049.

8 Even J, Pedesseau L, Katan C. Analysis of multivalley and multibandgap absorption and enhancement of free carriers related to exciton screening in hybrid perovskites. J Phys Chem C 2014; 118: 11566-11572.

9 Kulkarni SA, Baikie T, Boix PP, Yantara N, Mathews N et al. Band-gap tuning of lead halide perovskites using a sequential deposition process. J Mater Chem A 2014; 2: 9221-9225.

10 Mosconi E, Umari P, De Angelis F. Electronic and optical properties of mixed $\mathrm{Sn}-\mathrm{Pb}$ organohalide perovskites: a first principles investigation. J Mater Chem A 2015; 3: 9208-9215.

11 Katan C, Pedesseau L, Kepenekian M, Rolland A, Even J. Interplay of spin-orbit coupling and lattice distortion in metal substituted 3D tri-chloride hybrid perovskites. J Mater Chem A 2015; 3: 9232-9240.

12 Boix PP, Agarwala S, Koh TM, Mathews N, Mhaisalkar SG. Perovskite solar cells: beyond methylammonium lead iodide. J Phys Chem Lett 2015; 6: 898-907.

13 Amat A, Mosconi E, Ronca E, Quarti C, Umari P et al. Cation-induced band-gap tuning in organohalide perovskites: interplay of spin-orbit coupling and octahedra tilting. Nano Lett 2014; 14: 3608-3616

14 Koh TM, Fu KW, Fang Y, Chen S, Sum TC et al. Formamidinium-containing metalhalide: an alternative material for near-IR absorption perovskite solar cells. J Phys Chem C 2014; 118: 16458-16462.

15 Pang SP, Hu H, Zhang JL, Lv SL, Yu YM et al. $\mathrm{NH}_{2} \mathrm{CH}=\mathrm{NH}_{2} \mathrm{Pbl}_{3}$ : an alternative organolead iodide perovskite sensitizer for mesoscopic solar cells. Chem Mater 2014; 26: 1485-1491.

16 Wang $\mathrm{F}, \mathrm{Yu} \mathrm{H}, \mathrm{Xu} \mathrm{HH}$, Zhao N. $\mathrm{HPbl}_{3}$ : a new precursor compound for highly efficient solution-processed perovskite solar cells. Adv Funct Mater 2015; 25: 1120-1126.

17 Wozny S, Yang MJ, Nardes AM, Mercado CC, Ferrere S et al. Controlled humidity study on the formation of higher efficiency formamidinium lead triiodide-based solar cells. Chem Mater 2015; 27: 4814-4820.

18 Eperon GE, Stranks SD, Menelaou C, Johnston MB, Herz LM et al. Formamidinium lead trihalide: a broadly tunable perovskite for efficient planar heterojunction solar cells. Energy Environ Sci 2014; 7: 982-988. 
19 Jeon NJ, Noh JH, Yang WS, Kim YC, Ryu S et al. Compositional engineering of perovskite materials for high-performance solar cells. Nature 2015; 517: 476-480.

20 Yang WS, Noh JH, Jeon NJ, Kim YC, Ryu S et al. High-performance photovoltaic perovskite layers fabricated through intramolecular exchange. Science 2015; 348 1234-1237.

21 Fang HH, Raissa R, Abdu-Aguye M, Adjokatse S, Blake GR et al. Photophysics of organic-inorganic hybrid lead iodide perovskite single crystals. Adv Funct Mater 2015; 25: 2378-2385.

22 D'Innocenzo V, Grancini G, Alcocer MJP, Kandada ARS, Stranks SD et al. Excitons versus free charges in organo-lead tri-halide perovskites. Nat Commun 2014; 5: 3586.

23 Miyata A, Mitioglu A, Plochocka P, Portugall O, Wang JT-W et al. Direct measurement of the exciton binding energy and effective masses for charge carriers in organic-inorganic tri-halide perovskites. Nat Phys 2015; 11: 582-587.

24 Stoumpos CC, Malliakas CD, Kanatzidis MG. Semiconducting tin and lead iodide perovskites with organic cations: phase transitions, high mobilities, and near-infrared photoluminescent properties. Inorg Chem 2013; 52: 9019-9038.

25 Tanaka K, Takahashi T, Ban T, Kondo T, Uchida K et al. Comparative study on the excitons in lead-halide-based perovskite-type crystals $\mathrm{CH}_{3} \mathrm{NH}_{3} \mathrm{PbBr}_{3} \mathrm{CH}_{3} \mathrm{NH}_{3} \mathrm{Pbl}_{3}$. Solid State Commun 2003; 127: 619-623.

26 Lin QQ, Armin A, Nagiri RCR, Burn PL, Meredith P. Electro-optics of perovskite solar cells. Nat Photon 2015; 9: 106-112.

27 Yamada Y, Nakamura T, Endo M, Wakamiya A, Kanemitsu Y. Photoelectronic responses in solution-processed perovskite $\mathrm{CH}_{3} \mathrm{NH}_{3} \mathrm{Pbl}_{3}$ solar cells studied by photoluminescence and photoabsorption spectroscopy. IEEE J Photovolt 2015; 5: 401-405.
28 Wu KW, Bera A, Ma C, Du YM, Yang Y et al. Temperature-dependent excitonic photoluminescence of hybrid organometal halide perovskite films. Phys Chem Chem Phys 2014; 16: 22476-22481.

29 Vening M, Dunstan DJ, Homewood KP. Thermal quenching and retrapping effects in the photoluminescence of $\operatorname{In}_{y} \mathrm{Ga}_{1-\mathrm{y}} \mathrm{As} / \mathrm{GaAs} / \mathrm{Al}_{x} \mathrm{Ga}_{1-x} \mathrm{As}$ multiple-quantumwell structures. Phys Rev B 1993; 48: 2412-2417.

30 Grundmann M. The Physics of Semiconductors. Heidelberg: Springer-Verlag; 2010.

31 Gauthron K, Lauret JS, Doyennette L, Lanty G, Al Choueiry A et al. Optical spectroscopy of two-dimensional layered $\left(\mathrm{C}_{6} \mathrm{H}_{5} \mathrm{C}_{2} \mathrm{H}_{4}-\mathrm{NH}_{3}\right)_{2}-\mathrm{Pbl}_{4}$ perovskite. Opt Express 2010; 18 : 5912-5919.

32 Morello G, De Giorgi M, Kudera S, Manna L, Cingolani R et al. Temperature and size dependence of nonradiative relaxation and exciton-phonon coupling in colloidal CdTe quantum dots. J Phys Chem C 2007; 111: 5846-5849.

33 Hirasawa M, Ishihara T, Goto T. Exciton features in 0-, 2-, and 3-dimensional networks of $\left[\mathrm{PbI}_{6}\right]^{4-}$ Octahedra. J Phys Soc Jpn 1994; 63: 3870-3879.

(c) (1) (2) This work is licensed under a Creative Commons AttributionBY NC SA NonCommercial-ShareAlike 4.0 International License. The images or other third party material in this article are included in the article's Creative Commons license, unless indicated otherwise in the credit line; if the material is not included under the Creative Commons license, users will need to obtain permission from the license holder to reproduce the material. To view a copy of this license, visit http:// creativecommons.org/licenses/by-nc-sa/4.0/

Supplementary Information for this article can be found on the Light: Science \& Applications' website (http://www.nature.com/lsa) 